\title{
Design of the Integrated Intelligent Detection Platform for Electric Energy Measuring Device
}

\author{
CHENG Fu-yong ${ }^{1,2^{*}}$, CAO Min ${ }^{1,2}$, LIN Zhong-ai ${ }^{1,2}$, WANG Hong-lin ${ }^{1,2}$, CHEN Zheng ${ }^{1,2}$, LI Bo $^{1,2}$, and TANG Biao ${ }^{1,2}$
}

1. Yunnan Electric Power Research Institute, Kunming 65027, Yunnan Province, China;

2. Key Laboratory of CSG for Electric Power Measurement, Kunming 650217, Yunnan Province, China.

\begin{abstract}
For effectively detect and estimate the performance of various electric energy measuring devices, this paper proposes an integrated intelligent detection platform for the electric energy measuring device, which includes detection hardware, software and a good communication between hardware and software. With the study on the power system, communication circuit, voltage and current loop, data processing executive board and carrier communication, the hardware of integrated intelligent detection platform is designed. It also gives the software framework of detection platform and the design of information interaction for software and hardware. The integrated intelligent electric energy measuring device can greatly save time and improve detection efficiency.
\end{abstract}

\section{Introduction}

With the development of the power industry, various electric energy measuring devices are widely applied in power management systems [1-3]. So far, it is hard to detect different kinds of acquisition terminals for electric energy measuring devices. On the one hand, the product quality of acquisition terminal is quite different and may be poor, leading to difficulty on detection in a short time; on the other, the function of electric energy measuring devices is too unitary, which is unable to systematic detection. Therefore, it is promising to develop an effective and comprehensive detection platform [4-6].

Up to now, the detections of various electric energy measuring devices need different kinds of platforms and only can do some basic performances [7-8]. It is very difficult to compressively estimate the performance and communication of electric energy measuring devices. How to solve this issue has become a focal point in academia. Therefore, we should develop the integrated intelligent detection platform for measuring device including energy meter, data collator, concentrator, management terminal of distribution transformer and load control. The objective is to synthetic evaluate performance of the above measuring devices, which improves the efficiency of work.

This study aims to develop an integrated intelligent detection platform for different kinds of electric energy measuring devices or acquisition terminals. The proposed integrated intelligent detection platform includes detection hardware and software, and a good communication between hardware and software is achieved.

\section{The framework of integrated intelligent detection platform}

The framework of integrated intelligent detection platform includes a control cabinet and a wall-mounted table, as shown in Figure 1. The control cabinet is equipped with program-controlled three-phase power source CL303, power consumption tester CL2031, threephase multi-function standard table CL3115, serial server CL2018, precision time base source CL191B and $\mathrm{PC}$, which are controlled and managed by computer. The wall-mounted table is equipped with some terminals, such as current terminal, data processing execution board, control board, PT voltage isolator, carrier communication module, three-phase current terminal, voltage connection port, and multi-function pulse test port, etc. The wall-mounted table adopt integrated structure design, which can accommodate at least 8 three-phase multi-function meters and 2 metering terminals.

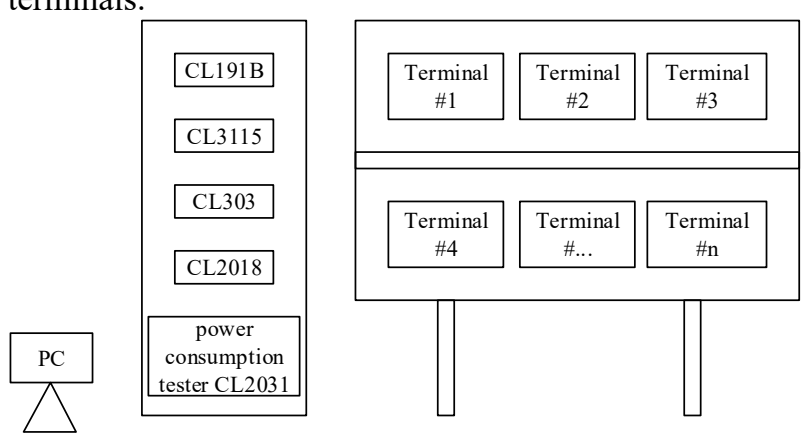

Fig. 1. The overall design of an integrated intelligent detection platform.

\footnotetext{
* Corresponding author: futao phd@163.com
} 
The characteristics of the integrated intelligent detection platform, as follows.

(1) The communication server is adopted, which is ensure that the functional tests of all epitopes are carried out simultaneously.

(2) Adopt RS-232 or RS-485, carrier wave, GSM/GPRS to communication with the terminals synchronous.

(3) Simulate various power consumption environments and electricity power stealing automatically.

(4) Detect the performance index of the power management terminals.

(5) Simulate various faults to detect whether all alarms are meet the set conditions.

(6) The programmable three-phase power source CL303 can output three-phase voltage, current and phase with high stability and accuracy.

(7) The wide-range three-phase multi-function standard table CL3115 can test the parameters such as voltage, current, phase, frequency and power.

(8) It has an emergency stop function in abnormal situation.

\section{The design of integrated intelligent detection platform}

In order to meet the needs of customers, the verification functions of electricity management terminals and electricity meters are integrated into one integrated intelligent detection platform, which can not only verify the functions of concentrator and collector, but also the functions of single/three-phase electricity meters. The integrated intelligent detection platform can perform parameter configuration and test on the collection terminal, and automatic detect the indicators of various functions for the collection terminals. The design of the platform mainly includes the design and implementation of hardware and software.

\subsection{Hardware design}

The hardware design of integrated intelligent detection platform fully meets the testing needs, and various situations to ensure the testing accuracy. It combines the functions of the energy meters test and the load control terminals test, which can detect both the energy meter and the concentrator/collector. The features are as follows.

(1) Compatible with electric energy meters and load control terminals.

(2) Using multi-channel server CL2018 to provide parallel protocol communication for all standard tables, sources, data processing execution boards, which greatly improving communication efficiency.

(3) Compatible with single-phase energy meters and three-phase energy meters.

(4) The design of current loop and voltage.

(5) The design of full-function data processing execution board.
The hardware of the detection device adopts modular design, which is includes the design of power supply system, communication circuit, voltage and current loop, data processing executive board and carrier communication.

\subsubsection{Power supply system}

The power system includes the power lines of control cabinet and wall-mounted table power. The power source CL303 of the control cabinet power supply uses a three-phase four-wire power, and the other instruments use $220 \mathrm{~V}$ power supply. The PT voltage isolator, carrier communication module, the detected terminals, and the power supply for the error board are powered by $220 \mathrm{~V}$. The power supply circuit can meet the power requirements of each instrument for sufficient demonstration, which ensure the stable and reliable operation and minimize the length of power supply line.

\subsubsection{Communication circuit}

(1) The overall communication system of integrated intelligent detection platform

The PC communicates with the multi-channel serial server CL2018-1 via TCP/IP protocol. The multichannel serial server CL2018-1 has 39 communication interfaces, which can realize the control of all the instruments of the detection platform, including the program-controlled three-phase Power source CL303, three-phase multi-function standard table CL3115, precision time base source CL191B-1, power consumption tester CL2013, error calculation board and execution board.

(2) Design of RS-485 communication system

Base on the multi-channel serial server CL2018, the RS485 communication network is arranged in the entire integrated intelligent test platform, so that the PC, power source, standard meter, power consumption tester, data processing execution board, and the detected terminal can communicate with each other in real time.

(3) 485 communication line design of wall-mounted table

The communication line has two main functions: the one is the communication between the CL2018 and the detected terminal/concentrator, and the other is the communication between the concentrator and the detected terminal. CL2018 communicates with the detected terminal through the 485 communication interface 2 when the PC gives an instruction for verifying function, and CL2018 communicates with the concentrator through the 485 communication interface 1 . The concentrator communicates with the detected terminal through the communication interface 1 after receiving the instruction.

\subsubsection{Voltage and current loop}

The integrated intelligent detection platform is compatible with the detection functions of single-phase electric energy meters and three-phase electric energy 
meters. When detecting the three-phase electric energy meters and acquisition terminals, the three-phase voltage is connected to the voltage terminals of the three-phase electric energy meters and acquisition terminals through a voltage connector. When detecting a single-phase meter, the voltage of the single-phase meter is directly taken from the live wire and neutral wire of current terminals, which is use the A-phase voltage of the threephase standard source, and then connected to the current terminal A and B after being separated by PT multichannel isolation transformer.

An extra relay is added in the B-phase current loop. When detecting three-phase meters, concentrators and collectors, the each position of wall-mounted table is connected to a relay by-pass, which the current loop is the same as that of the common watt-hour meter and electricity acquisition terminal.

\subsubsection{Data processing executive board}

The data processing execution board is the key of the integrated intelligent detection platform. Many verification items must be performed on the data processing execution board, such as energy measurement error, startup test, one-day time error, etc., to undertake error calculation, one-day time and remote control.

The data processing executive board of integrated intelligent detection platform can perform the function detection of all energy meters and collection terminals, and also detect remote control signals, output remote communication signal, and receive and process communication events.

According to different commands received by communication, the data processing executive board sends the order, which is applied to control the relay, remote signal output, terminal 485 switch, pulse output, power error calculation, one-day time error calculation, pulse calculation and other signal output. The data execution board software flow chart is shown in Figure 2.

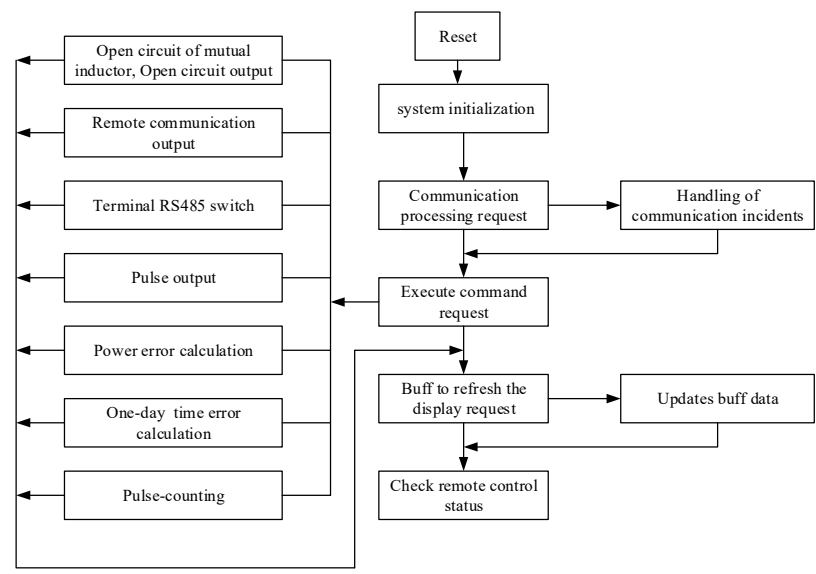

Fig. 2. The control flow diagram data processing executive board.

\subsubsection{Carrier communication}

The integrated intelligent detection platform is equipped with carrier communication test function. Compared with GPRS communication, 485 communication and small radio communication, carrier communication is no need to pay extra costs, nor the installation of communication lines.

Fig.3 shows the carrier signal receiving coupling circuit. The carrier signal (420KHZ) from the power line passes through the LC filter circuit, which can eliminate low-frequency signals (including $50 \mathrm{~Hz} \mathrm{AC}$ voltage). At the same time, the carrier signal will be located on the low voltage side passes through the isolation transformer. The bidirectional transient voltage suppressor suppresses the impulse voltage in the passband, and also suppresses the transient interference pulses and transient voltage on the power line. the isolation transformer plays the role of coupling, transmitting signals, and isolation, so that the power line circuit and the communication unit are safely isolated, thereby improving the signal anti-interference ability.

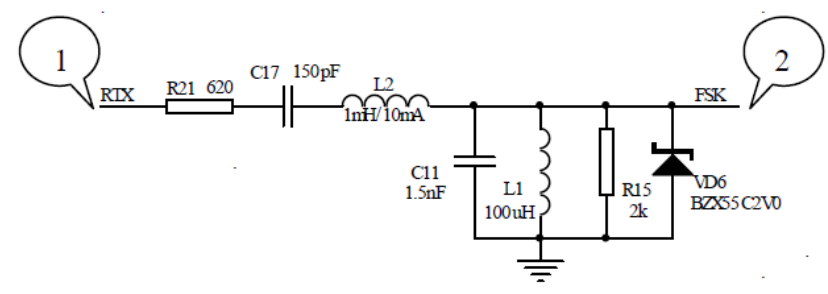

Fig. 3. The carrier signal receiving coupling circuit.

Fig.4 shows the carrier signal sending coupling circuit. The carrier signal sending circuit includes three parts: the input of spread spectrum modulation signal, a resonance power amplifier circuit and a feedback network circuit. The function of the carrier signal sending circuit is to output the signal power with high efficiency, and effectively inject the power of the spread spectrum modulation signal into the power line. The signal processed by the carrier chip is filtered and then amplified, and the amplified signal is sent back to the power line through the isolation transformer.

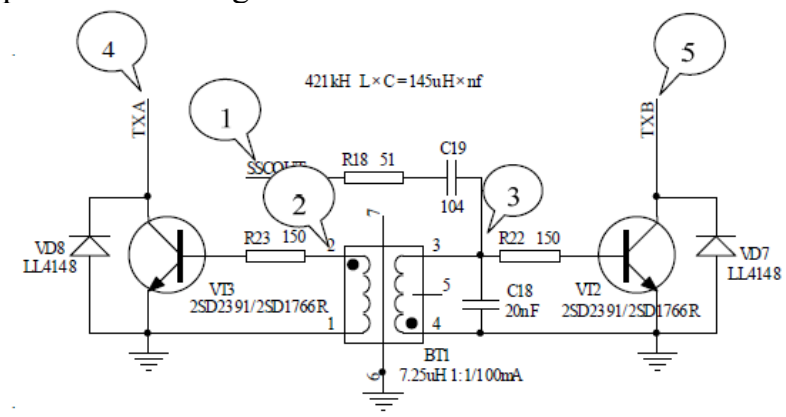

Fig.4. The carrier signal sending circuit

\subsection{Software design}

\subsubsection{The framework design of Software system}

Fig. 5 depicts the physical frame of integrated intelligent detection platform. It can unified managing the energy equipment and load control equipment. Additionally, it makes the hardware equipment with the same function possess one protocol. Therefore, the communication 
between the software system and the hardware of this platform is achieved by uniform protocols. This platform independently detects various electric managing equipment, such as load control terminals, concentrator, acquisition terminals and intelligent electric meter of reading data in simulation.

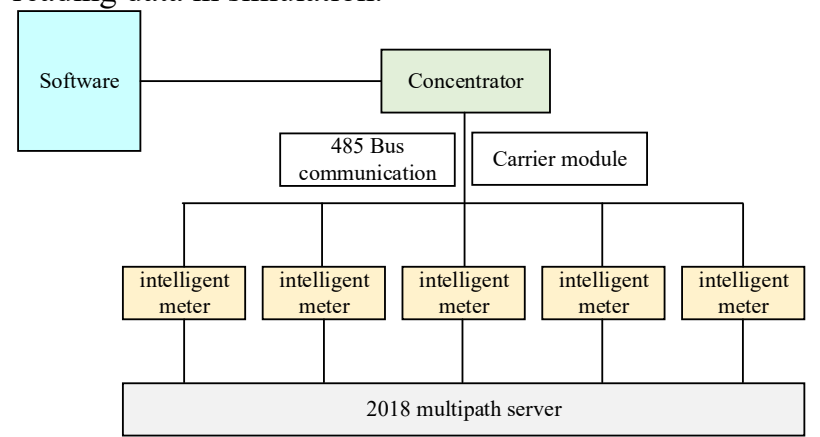

Fig. 5. Physical frame of intelligent detection platform.

Fig. 6 shows the software frame of integrated intelligent detection platform. The communication between the main program, control program, terminal and analog meter is achieved by the agreement of TCP. The multiple serial server is linked with the simulator of the electric meter and terminal by UDP. It is also connected with terminal by 485 . The software is composed of standalone program. The main program can exchange with data management and scheme management by data.

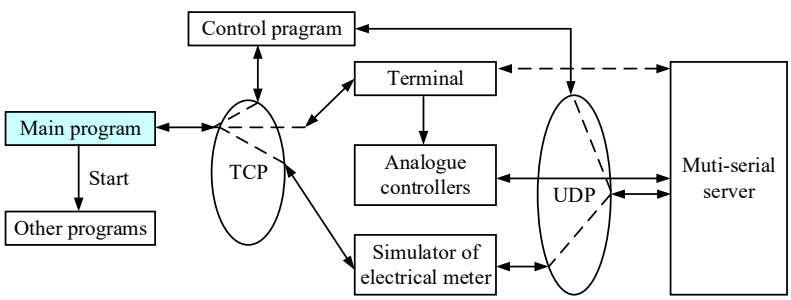

Fig. 6. Software frame of the intelligent detection platform.

\subsubsection{The information interaction for software and hardware}

The integrated intelligent detection platform is a large, complex, high-tech platform that contains both high-power components and precision devices. In order to realize the automatic detection function, good realtime communication is required between the $\mathrm{PC}$ and the instrument. The communication system of the integrated intelligent test platform is shown in Figure 7.

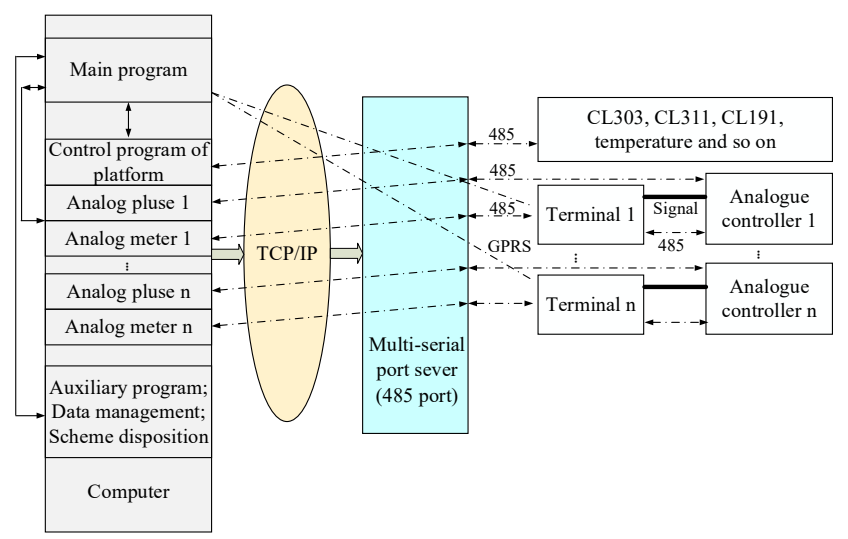

Fig.7. Communication system chart of the integrated intelligent detection platform.

The PC communicates with the multi-port server CL2018-1 through TCP/IP protocol. The multi-channel serial server CL2018-1 has 39 communication interfaces, which can control all the instruments of the integrated intelligent detection platform, including programcontrolled three-phase power source CL303, three-phase multi-function standard table CL3115, precision time base Source CL191B-1, power consumption tester CL2013, error calculation board and execution board.

The information interaction between software and hardware is through CL2018-1 parsing TCP/IP instructions, communicating with the source, standard instrument, terminal under test, and carrier communicator, which is to complete the interaction within the entire integrated test platform.

\subsubsection{The testing process of integrated intelligent detection platform}

Fig. 8 shows the testing process in detail for the proposed integrated intelligent detection platform. The specific steps are as follows.

(1) Write the parameter. Firstly, various function button including function keys of the interface need to work well. Then, the fault tolerance test is implemented for possibly wrong operation. In addition, the data integrity should be checked by the fault tolerance.

(2) Set system parameter. Firstly, the set parameters should be ensure correctness and can be saved. Then, the parameter of electric meter is set.

(3) Manage the data. Firstly, we check the different kinds of function buttons including the interface. Then, testers verify the appearance of report on data integrity.

(4) Manage the scheme. Firstly, the test items and sub-times of scheme on addition, deletion and duplication should be validated. Then, the verification about the default values of scheme need to carry out.

(5) Test the function. Firstly, single test function should be operated and cannot produce unexpected error. Then, automatic test of all functions is working properly.

(6) Conduct the comprehensive test. Firstly, the integrity of the whole program is tested. Also, the button function is checked and the fault tolerance test is conducted.

(7) Emulate the pulse of the electric meter. Firstly, it should make sure that the analog meter is only a 
subsidiary program when the main program is running. Then, the analog meter of the pulse is an independent program to finish testing work if the main program is closed.

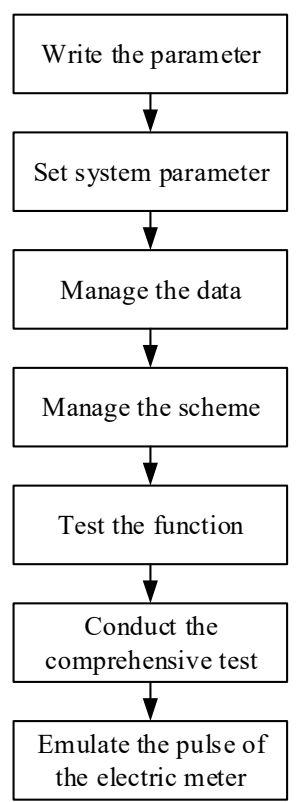

Fig.8. The testing process in detail for the proposed integrated intelligent detection platform

\section{Conclusion}

This paper mainly introduces for the hardware and software of the proposed integrated intelligent detection platform. For the design of hardware, it focuses on the power system, communication circuit, voltage and current loop, data processing executive board, and the carrier communication. For the design of software, it focuses on the architecture design, the communication system of software and hardware interaction, and the testing process.

The integrated intelligent detection platform contains a number of technological innovations. It is a powerful detection platform and represents the highest technical standard of the intelligent detection platform. Various types of tests can be carried out on the energy meters and the collection terminals. In the same location of the wallmounted table, it can detect both single-phase energy meters and three-phase energy meters, as well as collectors and concentrators. The integrated intelligent electric energy measuring device can greatly save time and improve detection efficiency.

\section{References}

1. S. B. Xia, S. Q. Wei, J. Wu. The Power of Information Collection Terminal Testing System. Instrumentation Technology. 2012,11:13-16.

2. C. H. Tang, G. Hu, J. Zhang, M. He. A New Centralized High Voltage Power Metering Terminal in Smart Substations. Automation of Electric Power System. 2011, 35(3):87-90.
3. H. C. Sun, L. Y. Zhang, W. F. Zhang, S. J. Wang. Research on the energy-controlling system for the terminal electric equipment in office building. Procedia Engineering. 2017, 205: 281-287.

4. L. Yang, D. Zhang, G. Lin, Q. Song, Q. Meng, Q. Pan, C. Liu. Research on remote calibration and online monitoring system of electric energy metering device. Journal of Physics: Conference Series. 2019, 1303(1): 012119.

5. A. Berouine, F. Lachhab, Y. N. Bakhouya, R. Ouladsine. A smart metering platform using big data and IoT technologies. 2017 3rd IEEE International Conference of Cloud Computing Technologies and Applications, 2017, pp. 1-6.

6. S. Huai, L. Yan, Z. Ang, Q. Song, Z. Wu. Research on technical architecture and application of big data cloud platform for electric power measurement. Journal of Physics: Conference Series. 2019, 1213(4): 042040.

7. S. L. Gong, Y. L. Wang, M. Y. Zhang. Design of intelligent power consumption optimization and visualization management platform for large buildings based on internet of things. MATEC Web of Conferences. 2017, 139: 00119.

8. P. Li. et al. Design of large-scale platform detection system based on distributed wireless communication technology. 2018 3rd Joint International Information Technology, Mechanical and Electronic Engineering Conference (JIMEC). 2018. 